\title{
Reanimation im Lehrplan der Schulen
}

\author{
Die Laienreanimation kommt in Deutschland zu kurz. Mit verschiedensten \\ Aktionen soll die Quote verbessert werden.
}

„Bessere Überlebenschancen durch schnelles Handeln", lautet eine Option gegen den plötzlichen Herztod. Eine Faustregel sagt, dass $50 \%$ der Patienten mit plötzlichem Herztod überleben bei sofortiger Herzmassage. Diese Rate sinkt mit jeder verstrichenen Minute ohne Herzmassage um $10 \%$. die Überlebenschance ist also nach fünf Minuten praktisch null.

Diese Zahlen begründen die Bedeutung der Laienreanimation vor Eintreffen des Notarztteams. Und hier zeigt sich Deutschland von seiner schwachen Seite. Während in Norwegen in 70 \% der Notfälle die Laienreanimation beginnt, bevor die Profihelfer eintreffen, beläuft sich diese Quote in den Niederlanden $63 \%$ und in Deutschland nur $17 \%$.

Diesen niederschmetternden Tatbestand können viele nicht nachvollziehen. Es werde doch schon viel getan, heißt es, immerhin müssen die Fahrschüler ErsteHilfe-Kurse besuchen, mit einem Zeitaufwand von 16 Stunden. Abgesehen davon, dass jedes ungenutzte Wissen mehr oder weniger lange Verfallszeiten hat, wächst mit den Jahren die Unsicherheit, wenn der ehemalige Fahrschüler im Ernstfall gefordert wird.

Deshalb sind Auffrischaktionen unerlässlich. Zahlreiche Einzelaktionen verschreiben sich diesem Ziel, sei es ein Gemeinschaftsprojekt der Herzstiftung mit

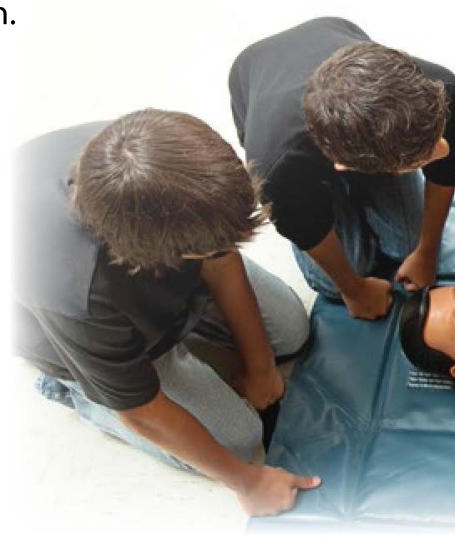

dem Deutschen Fußball-Bund zur Unterweisung von Altherrnspielern in der Laienwiederbelebung: „Lebensretter sein - Fußballer lernen Wiederbelebung“. Der Deutsche Rat für Wiederbelebung propagiert den Slogan „PrüfenRufen-Drücken“ und wirbt Mitglieder, um die Reanimationsversorgung in Deutschland zu verbessern.

Auf diesem Gebiet hat Prof. Wolfgang Schöls in Duisburg Pionierarbeit geleistet. Er hat mit Unterstützung der Herzstiftung und der Bürgerstiftung Duisburg zusammen mit Marketingprofis Spots nach modernen EntertainmentStandards entwickelt. Er bekam nach der Vorführung des Spots großen Applaus dafür. Sein Motto: „DU - ich drück' Dich!“ (auf Youtube zu sehen). Denn „Jeder muss drücken können“. Das Konzept: Die Anforderungen auf ein

\section{Nationales Aktionsbündnis Wiederbelebung}

Prof. Wolfgang Schöls vom Herzzentrum Duisburg stellte das Nationale Aktionsbündnis Wiederbelebung (NAWIB) vor, das vom Bundesgesundheitsministerium und der Bundeszentrale für gesundheitliche Aufklärung im September 2016 vorgestellt wurde und an der bislang 13 Fachgesellschaften mitarbeiten. Die Laienreanimationsquote soll nach Willen der Gründungsmitglieder, zu denen u.a. die DGK und Herzstiftung gehören, in Deutschland von derzeit $37 \%$ bis 2020 auf mindestens $50 \%$ gesteigert werden. Wichtig ist, an vielen Stellen anzusetzen, dazu zählen auch die Schulen. Deshalb wird das NAWIB die Schulen unterstützen, Wiederbelebung fest im Unterricht zu verankern. Der Schulausschuss der Kultusministerkonferenz hatte den Bundesländern bereits in seiner 395. Sitzung im Jahr 2014 empfohlen, ab der Jahrgangsstufe 7 zwei Unterrichtsstunden pro Jahr der Wiederbelebung zu widmen und die Lehrkräfte entsprechend schulen zu lassen. Mehrere Bundesländer haben die Empfehlung bereits umgesetzt, andere müssen nachziehen. Oftmals fehlt es aber an einem Ausbildungskonzept für Lehrer. Daher ist geplant, im Rahmen des NAWIB ein solches zu entwickeln.

Minimum senken, dafür flächendeckendes, kontinuierliches und nachhaltiges Training. Vorgesehen ist ein dreistufiges Prozedere:

_ Grundkonzept vermitteln, und zwar durch regelmäßige, wiederholte Anleitung im Schulunterricht. Ab der ersten Klasse im Sportunterricht; die Ausbildung der Sportlehrer inbegriffen.

- Selbstverständlichkeit verankern; Vorbild Verkehrserziehung durch „7. Sinn".

—Hemmungen abbauen, dazu werden frei zugängliche Trainingseinheiten im öffentlichen Raum vorgesehen. Schöls hat hierfür ein Gerät entwickelt, um die Brustkompression spielerisch zu trainieren.

\section{Cardiac-arrest-Zentren im Aufbau}

Auf klinisch-kardiologischer Seite ist man nicht untätig geblieben. Es werden „Cardiac arrest Zentren“-Konzepte diskutiert und auch realisiert, wie Prof. Andreas Schäfer von der Medizinischen Hochschule Hannover demonstrierte. In solchen Zentren, so Schäfer, „muss eine standardisierte Postreanimationsbehandlung überprüfbar garantiert sein“".

Das bedeutet: Standardisierter Behandlungspfad mit Einbindung aller nötigen Diagnostik und Therapie, Netzwerke zur intensivmedizinischen Frührehabilitation, Outcome-Assessment mit neurologischem Status und schließlich interdisziplinäre Aufarbeitung von Grenzsituationen.

Dr. med. Jochen Aumiller 